\title{
Global particle-in-cell simulations of fast-particle effects on shear Alfvén waves
}

\author{
Alexey Mishchenko* \\ Max-Planck-Institut für Plasmaphysik, \\ EURATOM-Association, D-17491 Greifswald, Germany \\ Axel Könies \\ Max-Planck-Institut für Plasmaphysik, \\ EURATOM-Association, D-17491 Greifswald, Germany \\ Roman Hatzky \\ Rechenzentrum der Max-Planck-Gesellschaft \\ und des Max-Planck-Instituts für Plasmaphysik, \\ EURATOM-Association, D-85748 Garching, Germany
}

(Dated: October 16, 2009)

\begin{abstract}
This paper reports self-consistent global linear gyrokinetic particle-in-cell simulations of shear Alfvén waves destabilized by fast particles in tokamak geometry. Resonant excitation of Toroidal Alfvén Eigenmodes by fast particles and their transition to Energetic Particle Modes (when the fast-particle drive is large enough) has been observed in the simulations.
\end{abstract}

* alexey.mishchenko@ipp.mpg.de 


\section{INTRODUCTION}

Fast particles can destabilize various shear Alfvén waves (SAW) [1]. These unstable perturbations are considered as the primary candidate for causing fluctuation-induced transport of the fast particles [2]. The destabilization of the SAW modes occurs if the mode drive resulting from interaction of a shear Alfvén wave with fast particles exceeds the mode damping which can be caused by various mechanisms, e.g. Landau damping, continuum damping [35], radiative damping [6-8] etc. It is known that weakly damped gap modes, such as Toroidal Alfvén Eigenmodes (TAE) [9], can be destabilized relatively easily [10] as long as they are not subjected to strong continuum damping [3,4]. If the fast-particle pressure is not too large, one can treat the TAE instability as a small perturbation of an ideal TAE mode. The frequency of this instability is a slightly modified ideal TAE frequency and the growth rate is small. There is a vast literature on the theory of the TAE instabilities, see e.g. Refs. [1012]. Numerically, the TAE modes have also been studied by many authors. For example, they have been simulated using the reduced kinetic approach [13], the first-principle global gyrokinetic eigenvalue approach $[14,15]$, the electron-fluid ion-kinetic hybrid gyrokinetic model $[16,17]$, and using the global gyrokinetic particle-in-cell (PIC) code [18].

When the pressure of the fast particles increases, the assumption that they cause only a small perturbation becomes invalid. In this regime, the physics is determined by nonperturbative phenomena, such as the Energetic Particle continuum Mode (EPM) [10, 19]. The drive of the EPM mode exceeds the stabilizing effect of the continuum damping so that the frequency of this mode can be inside of the SAW continuum. Both the frequency and the growth rate of the EPM mode are determined by the fast particles. The EPM modes have been studied analytically $[10,19]$ within the ballooning formulation using the multiple scale asymptotic technique. Numerically, the EPM instabilities have been simulated using the hybrid-MHD approach [20, 21]. In this approach, the background plasma is treated within the magnetohydrodynamic (MHD) theory whereas the fast-particle dynamics is gyrokinetic. The fast particles are coupled to the background through the fast-particle current or the fast-particle pressure which are computed as the corresponding moments of the fast-particle distribution function.

In the present paper, we use a global gyrokinetic particle-in-cell code GYGLES [18] to simulate both the unstable TAE modes and the EPM instabilities in a unified manner. 
All particle species (the bulk-plasma ions, the electrons, and the fast ions) are treated using gyrokinetic theory $[22,23]$. The finite-orbit-width effects are included in the global approach to all orders (there is no perturbative expansion with respect to the orbit width involved). The electrostatic and the parallel magnetic potentials are obtained from the quasineutrality equation and parallel Ampére's law. In our simulations, we observe a continuous modification of the TAE mode into the EPM instability caused by a change of the fast particle parameters (density or temperature).

The structure of the paper is as follows. Sec. II gives a short description of the basic equations and their discretization. Sec. III describes simulations of the TAE and the EPM instabilities. Final conclusions are drawn in Sec. IV.

\section{BASIC EQUATIONS AND NUMERICAL APPROACH}

The code solves the gyrokinetic Vlasov-Maxwell system of equations [22, 23]. The distribution function is split into the background part and the perturbation $f_{s}=F_{0 s}+\delta f_{s}$ (the index $s=i, e, f$ is used for the particle species: ions, electrons and fast particles). The background distribution function is usually taken to be a Maxwellian. The perturbed distribution function $\delta f_{s}$ is found from the linearized gyrokinetic Vlasov equation:

$$
\frac{\partial \delta f_{s}}{\partial t}+\dot{\mathbf{R}}^{(0)} \cdot \frac{\partial \delta f_{s}}{\partial \mathbf{R}}+\dot{v}_{\|}^{(0)} \frac{\partial \delta f_{s}}{\partial v_{\|}}=-\dot{\mathbf{R}}^{(1)} \cdot \frac{\partial F_{0 s}}{\partial \mathbf{R}}-\dot{v}_{\|}^{(1)} \frac{\partial F_{0 s}}{\partial v_{\|}}
$$

Here, $\left[\dot{\mathbf{R}}^{(0)}, \dot{v}_{\|}^{(0)}\right]$ correspond to the unperturbed gyrocenter position and parallel velocity. $\left[\dot{\mathbf{R}}^{(1)}, \dot{v}_{\|}^{(1)}\right]$ are the perturbation of the particle trajectories proportional to the electromagnetic field fluctuations. The equations of motion are:

$$
\begin{aligned}
\dot{\mathbf{R}} & =\left(v_{\|}-\frac{q}{m}\left\langle A_{\|}\right\rangle\right) \mathbf{b}^{*}+\frac{1}{q B_{\|}^{*}} \mathbf{b} \times\left[\mu \nabla B+q\left(\nabla\langle\phi\rangle-v_{\|} \nabla\left\langle A_{\|}\right\rangle\right)\right] \\
\dot{v}_{\|} & =-\frac{1}{m}\left[\mu \nabla B+q\left(\nabla\langle\phi\rangle-v_{\|} \nabla\left\langle A_{\|}\right\rangle\right)\right] \cdot \mathbf{b}^{*}
\end{aligned}
$$

with $\phi$ and $A_{\|}$being the perturbed electrostatic and magnetic potentials, $\mu$ the magnetic moment, $m$ the mass of the particle, $B_{\|}^{*}=\mathbf{b} \cdot \nabla \times \mathbf{A}^{*}, \mathbf{b}^{*}=\nabla \times \mathbf{A}^{*} / B_{\|}^{*}, \mathbf{A}^{*}=\mathbf{A}_{\mathbf{0}}+\left(m v_{\|} / q\right) \mathbf{b}$ the so-called modified vector potential, $\mathbf{A}_{\mathbf{0}}$ the magnetic potential corresponding to the equilibrium magnetic field $\mathbf{B}=\nabla \times \mathbf{A}_{\mathbf{0}}$ and $\mathbf{b}=\mathbf{B} / B$ the unit vector in the direction of the equilibrium magnetic field. The gyro-averaged potentials are defined as usual:

$$
\langle\phi\rangle=\oint \frac{\mathrm{d} \theta}{2 \pi} \phi(\mathbf{R}+\boldsymbol{\rho}),\left\langle A_{\|}\right\rangle=\oint \frac{\mathrm{d} \theta}{2 \pi} A_{\|}(\mathbf{R}+\boldsymbol{\rho})
$$


where $\boldsymbol{\rho}$ is the gyroradius of the particle and $\theta$ is the gyro-phase. The perturbed electrostatic and magnetic potentials are found self-consistently from the gyrokinetic quasineutrality equation and parallel Ampére's law:

$$
-\nabla \cdot\left[\left(\sum_{s=i, f} \frac{q_{s}^{2} n_{s}}{T_{s}} \rho_{s}^{2}\right) \nabla_{\perp} \phi\right]=\sum_{s=i, e, f} q_{s} \delta n_{s},\left(\sum_{s=i, e, f} \frac{\hat{\beta}_{s}}{\rho_{s}^{2}}-\nabla_{\perp}^{2}\right) A_{\|}=\mu_{0} \sum_{s=i, e, f} \delta j_{\| s},
$$

where $\delta n_{s}=\int \mathrm{d}^{6} Z \delta f_{s} \delta(\mathbf{R}+\boldsymbol{\rho}-\mathbf{x})$ is the perturbed gyrocenter density, $\delta j_{\| s}=$ $q_{s} \int \mathrm{d}^{6} Z \delta f_{s} v_{\|} \delta(\mathbf{R}+\boldsymbol{\rho}-\mathbf{x})$ is the perturbed gyrocenter current, $q_{s}$ is the charge of the particle, $\mathrm{d}^{6} Z=B_{\|}^{*} \mathrm{~d} \mathbf{R} \mathrm{d} v_{\|} \mathrm{d} \mu \mathrm{d} \theta$ is the phase-space volume, $\rho_{s}=\sqrt{m_{s} T_{s}} /(e B)$ is the thermal gyroradius and $\hat{\beta}_{s}=\mu_{0} n_{s} T_{s} / B_{0}^{2}$ is the "partial" plasma beta corresponding to a particular species. The polarization density is treated in the long-wavelength approximation. Finite Larmor radius (FLR) effects are neglected for electrons. The zeroth-order densities of the particle species satisfy the quasineutrality equation $\sum_{s} q_{s} n_{s}=0$ with $s=i, e, f$.

The perturbed part of the distribution function is discretized with markers:

$$
\delta f_{s}\left(\mathbf{R}, v_{\|}, \mu, t\right)=\sum_{\nu=1}^{N_{p}} w_{s \nu}(t) \delta\left(\mathbf{R}-\mathbf{R}_{\nu}\right) \delta\left(v_{\|}-v_{\nu \|}\right) \delta\left(\mu-\mu_{\nu}\right)
$$

where $N_{p}$ is the number of markers, $\left(\mathbf{R}_{\nu}, v_{\nu \|}, \mu_{\nu}\right)$ are the marker phase space coordinates and $w_{s \nu}$ is the weight of a marker. The electrostatic and magnetic potentials are discretized with the finite-element method (Ritz-Galerkin scheme):

$$
\phi(\mathbf{x})=\sum_{l=1}^{N_{s}} \phi_{l} \Lambda_{l}(\mathbf{x}), \quad A_{\|}(\mathbf{x})=\sum_{l=1}^{N_{s}} a_{l} \Lambda_{l}(\mathbf{x})
$$

where $\Lambda_{l}(\mathbf{x})$ are the finite elements (tensor product of B splines $[24,25]$ ), $N_{s}$ is the total number of the finite elements, $\phi_{l}$ and $a_{l}$ are the spline coefficients. A detailed description of the discretization procedure can be found in Refs. [26-30]. We apply the so-called phase factor transform [26] to all perturbed quantities in the code. The integrals over the gyroangle Eq. (4) are approximated with an N-point discrete sum [29, 31, 32]. The cancellation problem [27, 33] is solved using the iterative scheme No. 2 described in Ref. [30].

\section{SIMULATIONS}

We consider a large-aspect-ratio tokamak with a circular cross-section. The small radius is $r_{\mathrm{a}}=1 \mathrm{~m}$, the large radius is $R_{0}=10 \mathrm{~m}$, the magnetic field on the axis is $B_{0}=3 \mathrm{~T}$, the 
safety factor profile is $q(r)=1.71+0.16\left(r / r_{\mathrm{a}}\right)^{2}$ with $r$ being the minor radius of the plasma. The background magnetic field is $\mathbf{B}=\nabla \psi \times \nabla \varphi+I(\psi) \nabla \varphi$ with the toroidal angle $\varphi$, the poloidal flux $\psi$ and the poloidal current $I(\psi)$. In the large-aspect-ratio approximation, the poloidal current $I=B_{0} R_{0}$ and the poloidal flux $\psi(r)=B_{0} \int_{0}^{r}\left[r^{\prime} / q\left(r^{\prime}\right)\right] \mathrm{d} r^{\prime}$. The background plasma profiles are chosen to be flat with the ion density $n_{i}=2 \times 10^{19} \mathrm{~m}^{-3}$, the ion and electron temperatures $T_{i}=T_{e}=1 \mathrm{keV}$ (these parameters correspond to a bulk plasma with $\left.\beta=2 \mu_{0}\left(n_{i} T_{i}+n_{e} T_{e}\right) / B^{2} \approx 0.18 \%\right)$. The plasma consists of Hydrogen ions and electrons. The unperturbed ion and electron distribution functions are assumed to be Maxwellian.

We consider first the response of the background plasma in the absence of fast particles. The simulations are started initializing the perturbed electron distribution function with the toroidal mode number $n=-6$ and a poloidal spectrum which includes two harmonics with $m=10$ and $m=11$. The velocity dependence of the initial electron distribution function is Maxwellian. The initial perturbed ion distribution function is zero. For the safety factor chosen, there is a single gap in the SAW spectrum at $s=0.5$ where $q=1.75$ (see Fig. 1) as a result of the toroidicity-induced coupling of these harmonics. Here, $s=\sqrt{\psi / \psi_{\mathrm{a}}}$ with $\psi$ the poloidal flux and $\psi_{\mathrm{a}}$ the poloidal flux on the plasma edge. The radial dependence of the initial perturbed electron distribution function is chosen to be a Gaussian centered in the position of the gap. The time signal resulting from the PIC simulations is shown in Fig. 2. One sees that the poloidal modes with $m=10$ and $m=11$ are coupled to each other. The time signal measured has several spectral components (similarly to Ref. [18]). The dominant frequency, $\omega=0.413 \times 10^{6} \mathrm{rad} / \mathrm{s}$, agrees well with the ideal MHD frequency of the corresponding TAE mode $\omega_{\mathrm{MHD}}=0.414 \times 10^{6} \mathrm{rad} / \mathrm{s}$ (see Fig. 1). Also, the radial structures of the electrostatic and magnetic potentials (shown in Fig. 3) resemble a typical TAE pattern. Thus, the dominant part of the background plasma response to the initial perturbation applied is a stable TAE mode (the damping rate resulting from the PIC simulations is $\left.\gamma=-3.9 \times 10^{3} \mathrm{rad} / \mathrm{s}\right)$. More details regarding the PIC simulations of the stable Alfvénic modes in a tokamak geometry (such as the TAE mode considered here) can be found in Ref. [18]. In this paper, we will focus now on the self-consistent PIC simulations of shear Alfvén waves destabilized by fast particles.

We choose the unperturbed distribution of the fast particles (Deuterium ions) to be Maxwellian. The fast particle temperature $T_{f}$ is flat and the fast particle density is given 
by the expression:

$$
n_{f}(s)=n_{0 f} \exp \left[-\frac{\Delta_{\mathrm{n} f}}{L_{\mathrm{n} f}} \tanh \left(\frac{s-s_{\mathrm{n} f}}{\Delta_{\mathrm{n} f}}\right)\right]
$$

with $s_{\mathrm{n} f}=0.5$ the position of the maximal $\kappa_{\mathrm{n} f}=\left|\nabla n_{f}\right| / n_{f}, n_{0 f}$ the fast particle density at $s=s_{\mathrm{n} f}, \Delta_{\mathrm{n} f}=0.2$ the "width" of the density profile, and $L_{\mathrm{n} f}=0.3$ the "length" of the fast-particle density profile (this parameter determines how large the density gradient is).

We increase the value of the parameter $n_{0 f}$ stepwise. For the fast-particle density profile chosen (with $s_{\mathrm{n} f}=0.5$ ), the radial position of the maximal density gradient is $s=0.44$ and the radial position of the maximal $\kappa_{\mathrm{n} f}=\left|\nabla n_{f}\right| / n_{f}$ coincides with the position of the gap. Results of our simulations are summarized in Fig. 4, where the frequency and the growth rate are plotted as a function if of $n_{0 f}$. One sees that the TAE mode is destabilized by the fast particles at a certain threshold value of the fast-particle density. Further increasing the density leads to a continuous upward shift of the TAE frequency and the growth rate becomes larger. When the fast-particle density is large enough, the mode frequency leaves the gap and moves into the continuum, becoming an EPM instability (in Ref. [19] denoted a TAE-type Energetic Particle Mode). One sees that at the largest fast-particle density considered (the rightmost point on the figures), the frequency shift and the mode growth rate are comparable with the frequency of the ideal TAE (shown in Fig. 1). Note that the ratio of the fast-particle density to the bulk-plasma density is $n_{0 f} / n_{i} \approx 0.075$ and the ratio of the fast-particle beta (defined as $\beta_{f}=2 \mu_{0} n_{f} T_{f} / B^{2}$ ) to the bulk-plasma beta is $\beta_{f} / \beta \approx 30$ at this point. Of course, effects of the fast particles on the plasma equilibrium must be taken into the account at that large $\beta_{f}$ (such as the pressure anisotropy etc). This is, however, beyond the scope of the present paper.

On the right of Fig. 4, we compare the growth rate resulting from the PIC code with the growth rate obtained with a hybrid-MHD code $[34,35]$. It is clear that the perturbative hybrid-MHD approach is not valid when the fast-particle beta is larger than the bulkplasma beta. However, even when $\beta_{f}<\beta$ the growth rate obtained with the hybrid-MHD code is overestimated. The possible explanation is that the global hybrid-MHD approach $[34,35]$ lacks certain core-plasma damping mechanisms, such as the radiative damping or the continuum damping. We believe, however, that at low fast-particle beta the most important damping mechanism is due to the finite fast-particle gyroradius and finite orbit width effects. In what follows, we will see, for example, that the TAE modes become considerably more 
unstable if the fast particles are driftkinetic. Thus, an incomplete description of the mode damping leads to overestimated growth rates in the hybrid-MHD approach [34, 35].

In Fig. 5, the radial structure of the EPM mode resulting from our simulations is shown (here for $n_{0 f}=10^{18} \mathrm{~m}^{-3}$ ). One sees that the poloidal mode structure of this EPM instability is similar to the TAE mode shown above, although the radial width of the EPM mode is considerably larger. Besides that, the radial location of the EPM mode maximum is shifted towards the position of the maximal fast-particle density gradient $s=0.44$ (in contrast with the TAE mode Fig. 3 whose maximum is radially located in the position of the gap $s=0.5$ ).

It is interesting to study parametric dependencies of the modes which are not so strongly destabilized by the fast particles, because this case may be more relevant in practice. For this purpose, we take the marginally unstable TAE mode with $n_{0 f}=0.75 \times 10^{17} \mathrm{~m}^{-3}$ and $T_{f}=0.4 \mathrm{MeV}$. We change the temperature of the fast ions, keeping their density constant. The result of these simulations is shown in Fig. 6. One sees that the mode frequency is shifted downward at small fast-particle temperatures and increases when the fast-particle temperature increases but does not leave the gap (the TAE mode does not become an EPM instability). The growth rate is a non-monotonic function of the fast-particle temperature. One can explain this taking into account that the TAE destabilization occurs due to a resonance interaction of the fast particles with the wave. This interaction is most effective at a certain energy of the fast particles. Changing the temperature, one changes the number of the fast particles satisfying the resonance condition. In addition, the growth rate of the TAE mode depends on the fast-particle pressure (it increases with the pressure). Clearly, the fast-particle pressure is larger at larger temperatures so far the fast-particle density is kept constant. On the other hand, at larger temperatures stabilizing finite-orbit-width (FOW) effects [36] may become more important. In Fig. 6 (on the right), we compare the growth rate resulting from the PIC code including all FLR and FOW effects (solid line, circles) with the growth rate (dotted line, diamonds) obtained with the PIC code using the driftkinetic fast particles (i.e. without the fast-particle FLR effects). Also, the growth rate obtained with the hybrid-MHD approach $[34,35]$ where only the electron Landau damping has been included is shown (dashed line, no symbols). One sees that the results of the PIC simulations without the fast-particle FLR effects agree well with the hybrid-MHD results if the fast-particle temperature is not too large. At the larger fast-particle temperatures, the orbit width of the fast particles increases and may become comparable to the TAE width. 
For example, at $T_{f}=300 \mathrm{keV}$, the fast-particle orbit shift from magnetic flux surface $\delta_{f} \approx q(R / r)^{1 / 2} \rho_{f} \approx 15 \mathrm{~cm}$ which is approximately one half of the TAE mode radial width. Thus, one can make the conjecture (following Ref. [36]) that the FOW effects make the TAE mode less unstable. However, it is difficult to prove this directly (as it has been done in Ref. [36]) within the PIC initial-value framework.

Consider now how the growth rate changes with the fast-particle temperature if the fast-particle pressure is kept constant (instead of the fast-particle density). We choose it at the value corresponding to $n_{0 f}=0.75 \times 10^{17} \mathrm{~m}^{-3}$ and $T_{f}=0.4 \mathrm{MeV}$. The fastparticle beta $\beta_{f} \approx 0.134 \%$ for these parameters which corresponds to $\beta_{f} / \beta \approx 0.75$ with $\beta \approx 0.18 \%$ the bulk-plasma beta. Similarly to Fig. 6, the growth rate in Fig. 7 is a nonmonotonic function of the fast-particle temperature. It is maximal at the temperature where the resonant parallel velocity $v_{\|(\text {res })}=v_{\mathrm{A}} / 3$ (see Ref. [10]) is close to the thermal one (here, $v_{\mathrm{A}}=B / \sqrt{\mu_{0} m_{i} n_{i}}$ is the Alfvén speed). This resonance condition gives the temperature $T_{f(\mathrm{res})} \approx m_{\mathrm{D}} v_{\|(\mathrm{res})}^{2} / 2 \approx 0.226 \mathrm{MeV}\left(m_{\mathrm{D}}\right.$ is the Deuterium mass) which corresponds indeed to the maximum of the growth rate in Fig. 7. The growth rate may decrease at larger temperatures due to FOW effects [36] $\left(k_{\perp} \rho_{f} \approx 1\right.$ at $T_{f}=0.8 \mathrm{MeV}$ where $\rho_{f}$ is the fastparticle gyroradius). This may explain why the resonance at $v_{\|(\text {res })} \approx v_{\mathrm{A}}$ (see Ref. [10]) is not so prominent because it is possible that the FOW effects quench the mode drive at the larger $k_{\perp} \rho_{f}$. In Fig. 6, this mode stabilization (which may be caused by the FOW effects) was actually less evident because there $\beta_{f}$ increased linearly with $T_{f}$ (the fast-particle density was kept constant). The mode frequency does not change much at larger fast-particle temperatures and is close to the frequency of the ideal TAE. The mode structure coincides with that of a conventional TAE (see Fig. 8 for the case with $T_{f}=0.6 \mathrm{MeV}$ ).

At smaller fast-particle temperatures, the mode frequency leaves the gap (see Fig. 7). Note that the fast-particle density increases when the fast-particle temperature drops because the fast-particle pressure is kept constant. For example, the fast-particle density $n_{0 f}=6 \times 10^{17} \mathrm{~m}^{-3}$ at $T_{f}=50 \mathrm{keV}$ which corresponds to $n_{0 f} / n_{i}=0.03$. One sees that at these relatively large densities, the mode becomes a non-perturbative EPM instability. We dub this EPM instability "a type II mode" because it differs from the EPM mode (type I) which has been found previously (see Fig. 5). The EPM "type-II" mode (shown in Fig. 9 for the case with $T_{f}=0.05 \mathrm{MeV}$ ) has a single dominant poloidal mode ( $\left.m=10\right)$. Radially, it is localized near the position $s=0.35$ where the continuum frequency corresponding to $m=10$ 
matches the frequency $\omega_{\mathrm{EPM}}=0.33 \times 10^{6} \mathrm{rad} / \mathrm{s}$ of the EPM mode observed (cf. Fig. 1). The mode is quite narrow. When the temperature increases, the mode frequency increases as well. The mode shifts radially towards the gap position (moving "along" the continuum) and becomes wider. Note that the perturbative calculations [34, 35] cannot be used for any of the modes shown in Fig. 7. They are invalid at lower fast-particle temperatures (large $n_{0 f}$ ) because of the non-perturbative effects. At higher fast-particle temperatures, they overestimate the mode growth rate, possibly because of the FOW effects.

To consider how the growth rate of the TAE modes depends on the fast-particle density gradient, we change the parameter $L_{\mathrm{n} f}$ in Eq. (8). For this study, we take the case with the fast-particle density $n_{0 f}=1.5 \times 10^{17} \mathrm{~m}^{-3}$ and the fast-particle temperature $T_{f}=0.2 \mathrm{MeV}$ (this case corresponds to the maximum of the growth rate in Fig. 6, i.e. to the point where the $v_{\mathrm{A}} / 3$-resonance is most effective). One would expect that the mode is more unstable at larger density gradients. This indeed happens in our simulations as one can see in Fig. 10. In contrast with the growth rate, which changes substantially with the density gradient length, the frequency remains nearly constant (and stays in the gap). Despite the very similar frequencies, there is a difference between the mode at the largest density gradient (the largest gradient in the simulations shown in Fig. 10 corresponds to $1 / L_{\mathrm{n} f}=5.0$ ) and the mode at the smallest density gradient (the simulation with $1 / L_{\mathrm{n} f}=2.5$ ). In Fig. 11 , one sees that the mode at the smaller density gradient is localized around the position of the gap $s=0.5$ whereas the mode at the larger density gradient is wider and is radially shifted towards the position of the maximal fast-particle density gradient $s=0.44$. It seems that the mode at the larger fast-particle density gradient is non-perturbative (it has similarities with the EPM "type-I" instability which we have observed previously in Fig. 5) although the frequency of this mode is in the gap.

We now consider if the results of our simulations are well converged with respect to various numerical parameters. In Fig. 12, a convergence study with respect to the marker resolution is shown. Although the frequency and the growth rate are shown as function of the number of the ion markers, we have increased simultaneously (in proportion) the number of the markers of all the species (ions, electrons and fast particles). Thus, the first point corresponds to $N_{i}=1.25 \times 10^{5}$ ion markers, $N_{e}=5 \times 10^{5}$ electron markers and $N_{f}=2.5 \times 10^{5}$ fast-particle markers. The second point has been obtained using $N_{i}=2.5 \times 10^{5}, N_{e}=10^{6}$, $N_{f}=5 \times 10^{5}$, and so on. Excluding the very first point on the both graphs, the relative 
deviation in the frequency is $\sim 0.1 \%$ and the relative deviation in the growth rate is $\sim 1 \%$. One sees that the simulations are well converged with respect to the marker resolution.

In Fig. 13, the convergence study with respect to the grid resolution is plotted. Here, we keep the number of markers per grid cell constant (meaning that the total marker number has to increase with the number of the radial grid points $N_{r}$ ). For $N_{r}=32$, we take $N_{i}=0.6 \times 10^{6}, N_{e}=2.4 \times 10^{6}$ and $N_{f}=1.2 \times 10^{6}$. One sees that the simulations are convergent with respect to the radial resolution, too.

Finally in Fig. 14, we study the convergence of our simulations with respect to the number of iterations [30] used to achieve the cancellation in Ampére's law [27, 33]. In all our simulations shown above, we have used $N_{\text {iter }}=4$. One sees that this number corresponds

to a well-converged situation. One sees also that too small $N_{\text {iter }}=1$ results in the wrong frequency and growth rate. The simulation without the iterative approach to the cancellation in Ampére's law $\left(N_{\text {iter }}=0\right)$ has been numerically unstable. This demonstrates that an accurate cancellation of the skin term and the adiabatic current [27, 33] in Ampére's law is crucial for the success of the electromagnetic PIC simulations.

\section{CONCLUSIONS}

In conclusion, we have presented global gyrokinetic PIC simulations of the shear Alfvén waves destabilized by fast particles in tokamak geometry. In our simulations, we have observed a stabilizing influence of the fast-particle FLR effects and, possibly, the fast-particle FOW effects on the TAE instability (see Fig. 6). A continuous modification of the unstable TAE mode into the EPM mode caused by a change of the fast particle parameters (density or temperature) has been observed, too. We have found that the mode becomes non-perturbative (EPM) when the fast-particle density is large enough. In our simulations, one can distinguish between the type-I EPM instability, which has been found when the fastparticle temperature is large (see Fig. 4), and the type-II EPM instability which appears when the fast-particle temperature is relatively small (but the density is still sufficiently high), such as in Fig. 7. The type-I EPM has a global radial structure and a poloidal spectrum similar to a conventional TAE. The type-II EPM has a single dominant poloidal mode. It is radially localized near the position where the SAW continuum frequency corresponding to the dominant poloidal mode number matches the frequency of the EPM mode observed. 
From the numerical point of view, we have seen that a moderate number of markers and a moderate radial resolution are sufficient to resolve the linear single-gap modes considered in this paper. However, we have found that the cancellation in Ampére's law must be accurate enough in order to succeed with the electromagnetic PIC simulations.

Looking ahead, there are plenty of topics which may be addressed with the global electromagnetic gyrokinetic PIC approach. The physics at the low-frequency part of the SAW spectrum is one such topic. At low frequencies, the bulk-plasma kinetic effects become important [37, 38] (because the core diamagnetic frequency becomes comparable to the frequency of the mode). A first-principle non-perturbative gyrokinetic PIC approach seems to be an appropriate tool for addressing this regime.

Recently, PIC simulations of fast-particle destabilized Alfvén modes similar to those presented in this paper have been performed with the GTC code [17] using an electronfluid ion-kinetic hybrid gyrokinetic model. Obviously, this reduced model is limited to the cases when the electron kinetic effects are unimportant (it can not be used to study the microtearing mode, for example). However, in contrast with the fully gyrokinetic approach, the cancellation problem does not appear when the electrons are treated as a fluid. Thus, it remains an interesting task for future to compare the full gyrokinetic PIC approach with the electron-fluid ion-kinetic hybrid gyrokinetic model both from the point of view of the physics included and the complexity of the computations required.

\section{ACKNOWLEDGMENTS}

We thank P. Helander and S. Günter for carefully reading the manuscript. We appreciate discussions with $\mathrm{Ph}$. Lauber and comments of A. Bottino. The computations have been done on the HGW Linux cluster (technical support from H. Leyh and M. Borchardt is acknowledged) and on the IBM Blue Gene/P supercomputer at the Rechenzentrum der Max-Planck-Gesellschaft und des Max-Planck-Instituts für Plasmaphysik.

[1] M. N. Rosenbluth and P. H. Rutherford, Phys. Rev. Lett. 34, 1428 (1975).

[2] F. Zonca, S. Briguglio, L. Chen, C. Fogaccia, T. S. Hahm, A. V. Milovanov, and G. Vlad, 
Plasma Phys. Controlled Fusion 48, B15 (2006).

[3] F. Zonca and L. Chen, Phys. Rev. Lett. 68, 592 (1992).

[4] H. L. Berk, J. W. V. Dam, Z. Guo, and D. M. Lindberg, Phys. Fluids B 4, 1806 (1992).

[5] M. N. Rosenbluth, H. L. Berk, J. W. Van Dam, and D. M. Lindberg, Phys. Fluids B 4, 2189 (1992).

[6] R. R. Mett and S. M. Mahajan, Phys. Fluids B 4, 2885 (1992).

[7] H. L. Berk, R. R. Mett, and D. M. Lindberg, Phys. Fluids B 5, 3969 (1993).

[8] J. Candy and M. N. Rosenbluth, Phys. Plasmas 1, 356 (1994).

[9] C. Z. Cheng, L. Chen, and M. S. Chance, Ann. Phys. 161, 21 (1985).

[10] L. Chen, Phys. Plasmas 1, 1519 (1994).

[11] G. Y. Fu and J. W. V. Dam, Phys. Fluids B 1, 1949 (1989).

[12] J. Candy and M. N. Rosenbluth, Plasma Phys. Controlled Fusion 35, 957 (1993).

[13] G. Y. Fu, H. L. Berk, and A. Pletzer, Phys. Plasmas 12, 082505 (2005).

[14] P. Lauber, S. Günter, and S. D. Pinches, Phys. Plasmas 12, 122501 (2005).

[15] P. Lauber, S. Günter, A. Könies, and S. D. Pinches, J. Comp. Phys. 226, 447 (2007).

[16] Y. Nishimura, Z. Lin, and W. X. Wang, Phys. Plasmas 14, 042503 (2007).

[17] Y. Nishimura, Phys. Plasmas 16, 030702 (2009).

[18] A. Mishchenko, R. Hatzky, and A. Könies, Phys. Plasmas 15, 112106 (2008).

[19] F. Zonca and L. Chen, Phys. Plasmas 7, 4600 (2000).

[20] Y. Todo, Phys. Plasmas 13, 082503 (2006).

[21] S. Briguglio, G. Fogaccia, G. Vlad, F. Zonca, K. Shinohara, M. Ishikawa, and M. Takechi, Phys. Plasmas 14, 055904 (2007).

[22] T. S. Hahm, Phys. Fluids 31, 2670 (1988).

[23] T. S. Hahm, W. W. Lee, and A. J. Brizard, Phys. Fluids 31, 1940 (1988).

[24] C. de Boor, A Practical Guide to Splines (Springer-Verlag, New York, 1978).

[25] K. Höllig, Finite Element Methods with B-Splines (Society for Industrial and Applied Mathematics, Philadelphia, 2003).

[26] M. Fivaz, S. Brunner, G. de Ridder, O. Sauter, T. M. Tran, J. Vaclavik, L. Villard, and K. Appert, Comp. Phys. Commun. 111, 27 (1998).

[27] A. Mishchenko, R. Hatzky, and A. Könies, Phys. Plasmas 11, 5480 (2004).

[28] A. Mishchenko, A. Könies, and R. Hatzky, in Proceedings of the Joint Varenna-Lausanne 
International Workshop, Varenna, 2004, edited by J. W. Connor, O. Sauter, and E. Sindoni (Società Italiana di Fisica, Bologna, 2004), p. 315.

[29] A. Mishchenko, A. Könies, and R. Hatzky, Phys. Plasmas 12, 062305 (2005).

[30] R. Hatzky, A. Könies, and A. Mishchenko, J. Comp. Phys. 225, 568 (2007).

[31] W. W. Lee, J. Comp. Phys. 72, 243 (1987).

[32] R. Hatzky, T. M. Tran, A. Könies, R. Kleiber, and S. J. Allfrey, Phys. Plasmas 9, 898 (2002).

[33] Y. Chen and S. Parker, J. Comp. Phys 189, 463 (2003).

[34] A. Könies, Phys. Plasmas 7, 1139 (2000).

[35] A. Könies, A. Mishchenko, and R. Hatzky, in Proceedings of the Joint Varenna-Lausanne International Workshop, Varenna, 2008, edited by X. Garbet, O. Sauter, and E. Sindoni (AIP Conference Proceedings, New York, 2008), Vol. 1069, p. 133.

[36] N. N. Gorelenkov, C. Z. Cheng, and G. Y. Fu, Phys. Plasmas 6, 2802 (1999).

[37] F. Zonca, L. Chen, J. Q. Dong, and R. Santoro, Phys. Plasmas 6, 1917 (1999).

[38] F. Zonca, L. Chen, and R. Santoro, Plasma Phys. Controlled Fusion 38, 2011 (1996). 


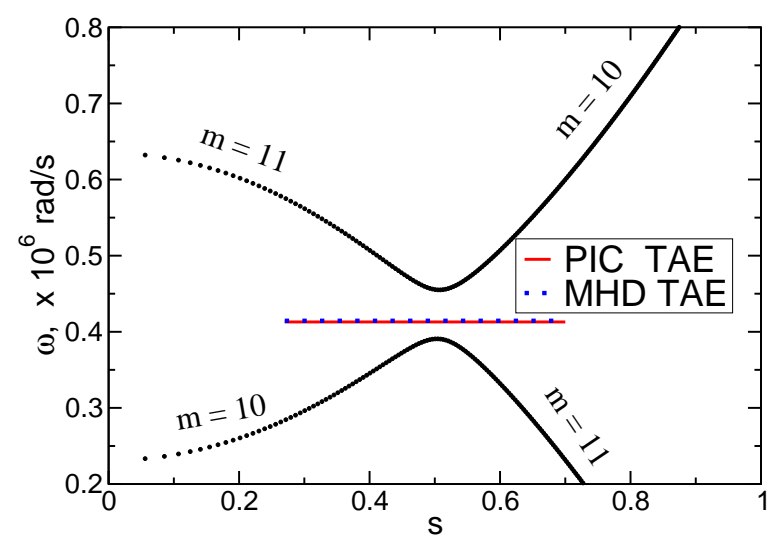

FIG. 1: (Color online) The shear Alfvén spectrum for the case considered (parameters see in the main text). The SAW continuum is plotted. The dominant frequency resulting from the PIC simulation (the stable TAE mode) is compared with the TAE mode computed with the ideal MHD eigenvalue code. 

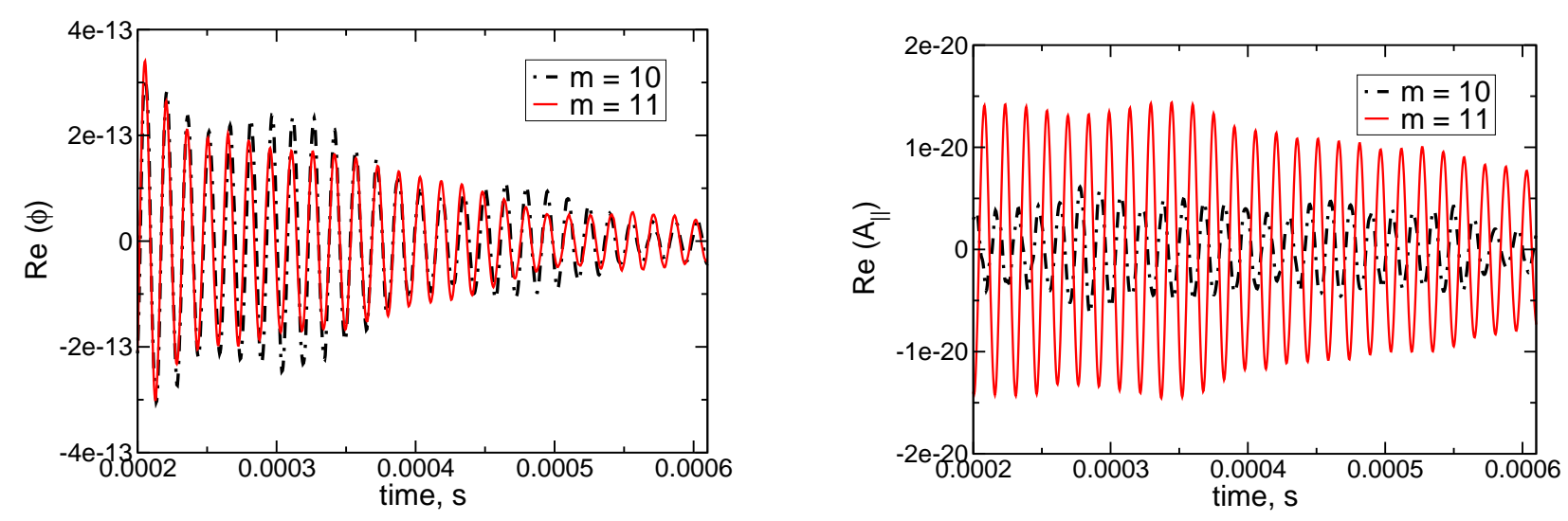

FIG. 2: (Color online) Time dependency of the electrostatic (left) and magnetic (right) potentials measured with the PIC code. One sees that the poloidal modes are coupled with each other. 

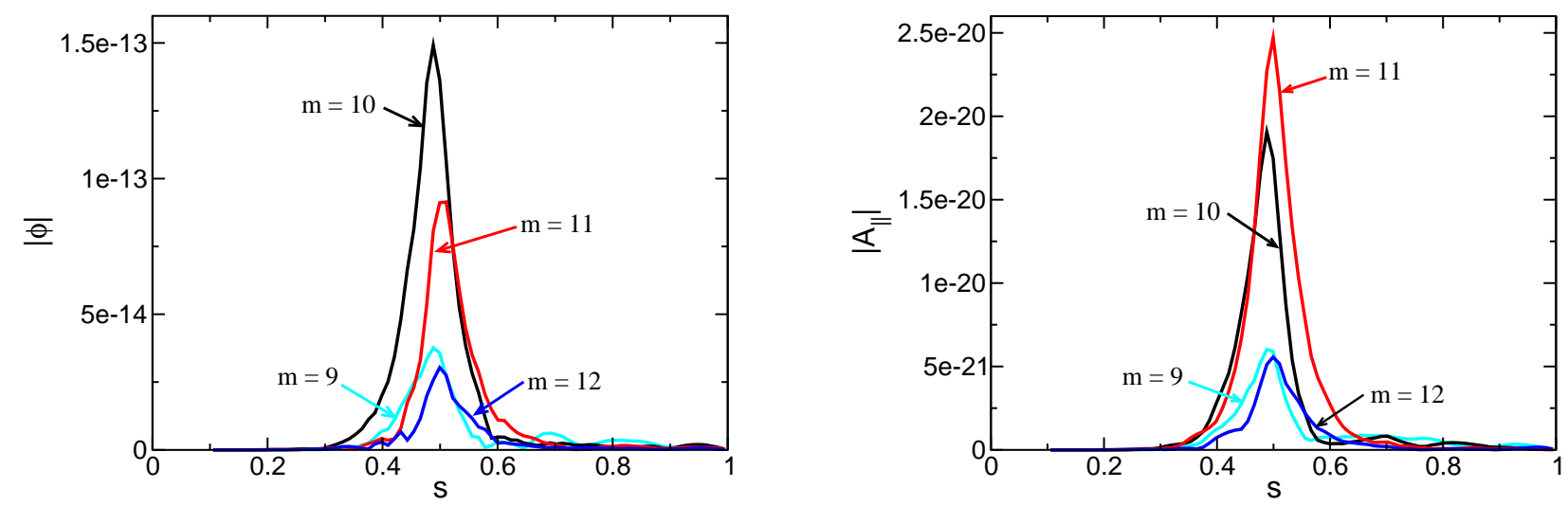

FIG. 3: (Color online) Radial structures of the electrostatic (left) and magnetic (right) potentials measured with the PIC code. One sees that the mode is centered on the gap position and has a TAE-type poloidal spectrum. 

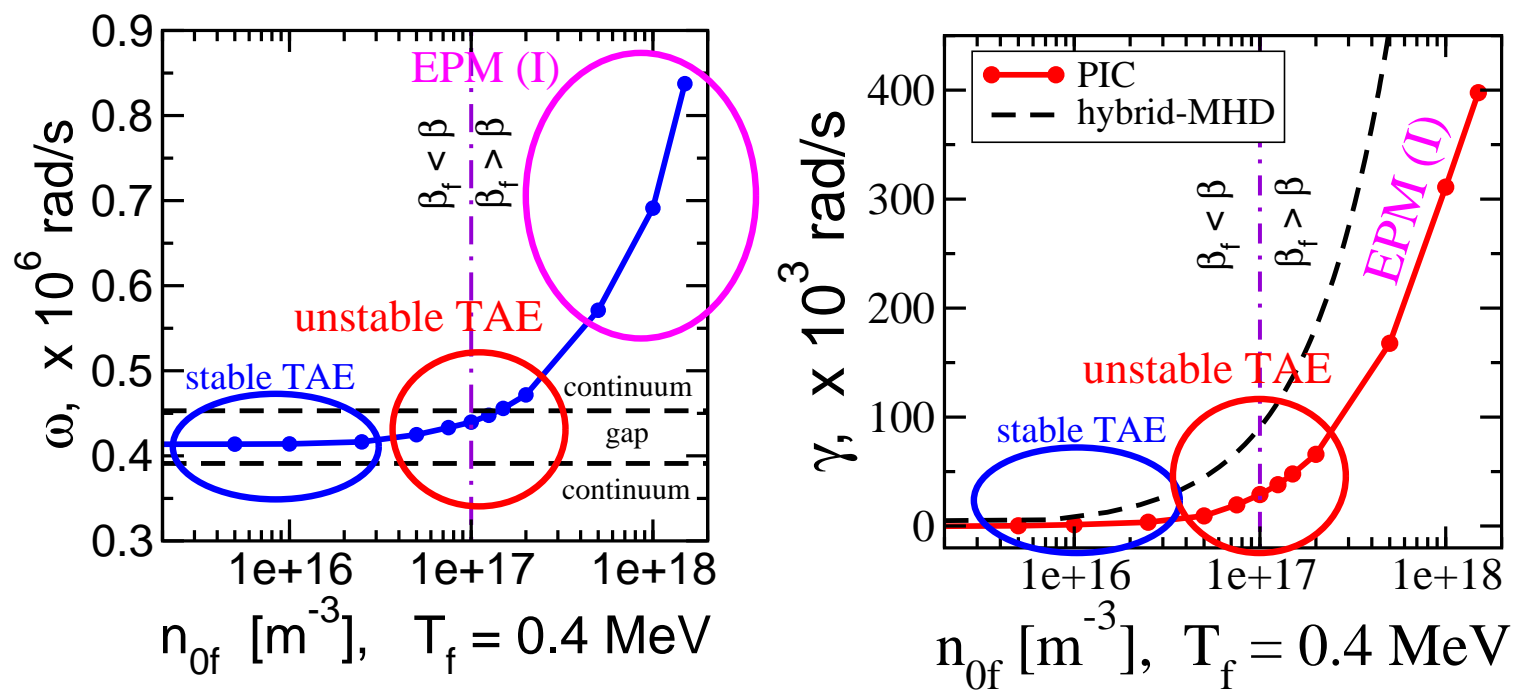

FIG. 4: (Color online) The frequency (left) and the growth rate (right) of the TAE mode destabilized by the fast particles (sweep over the fast particle density, the fast particle temperature is kept constant). The TAE mode transforms continuously into the EPM instability as $n_{0 f}$ increases. On the right, the PIC growth rate (solid line) is compared with the growth rates resulting from the hybrid-MHD approach $[34,35]$ (dashed line). For parameters see the main text. 

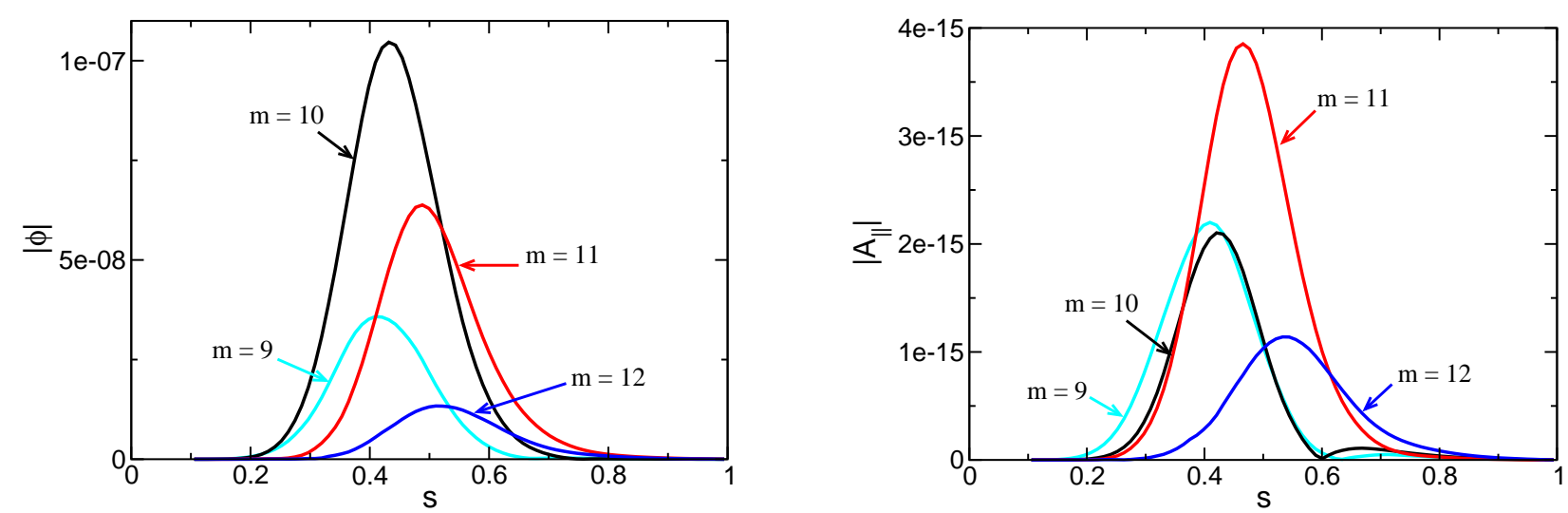

FIG. 5: (Color online) Radial structures of the electrostatic (left) and magnetic (right) potentials measured with the PIC code for the EPM mode corresponding to $n_{0 f}=10^{18} \mathrm{~m}^{-3}$ (this mode can be dubbed a TAE-type EPM similar to Ref. [19]). This EPM mode has a poloidal spectrum similar to the TAE mode but the radial width is much larger than that of a typical TAE mode such as shown in Fig. 3. 

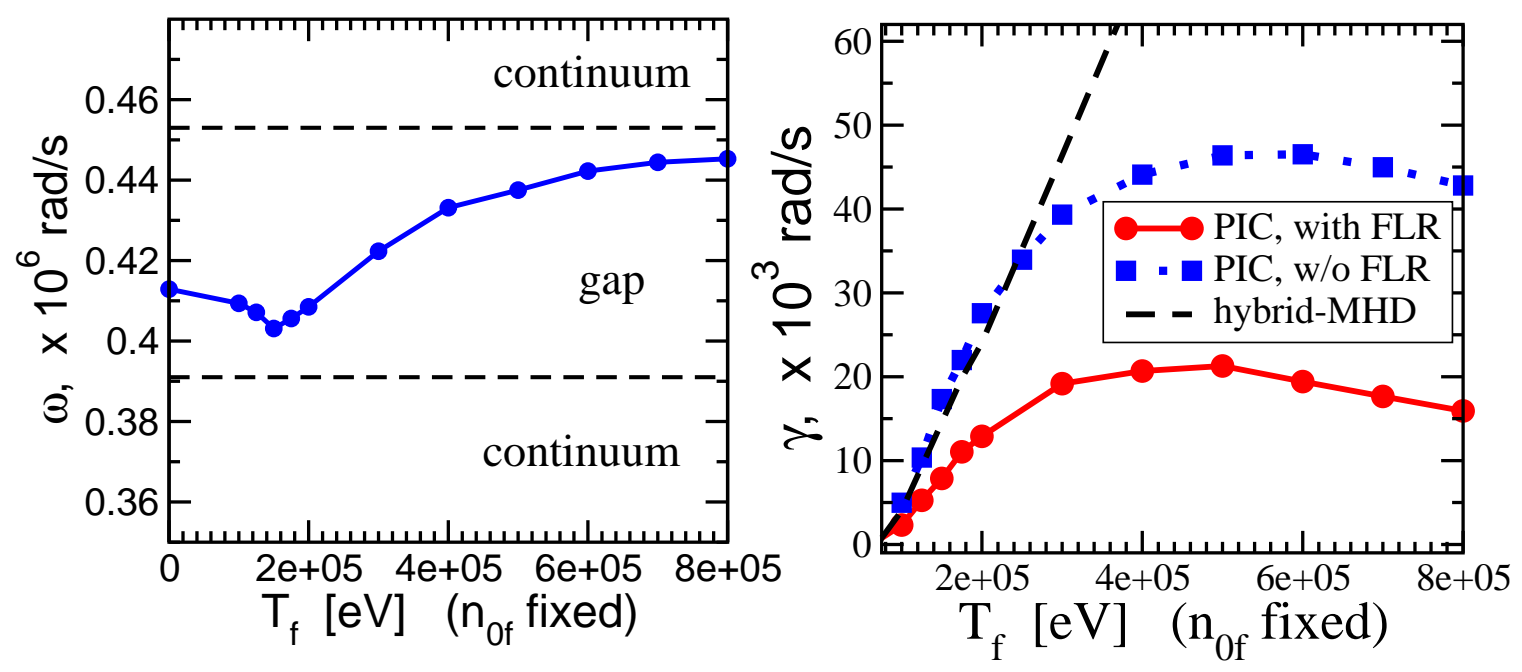

FIG. 6: (Color online) The frequency (left) and the growth rate (right) of the TAE mode destabilized by the fast particles (sweep over the temperature of the fast particles, $n_{0 f}=0.75 \times 10^{17} \mathrm{~m}^{-3}$ is kept constant). On the right, the PIC growth rate which includes all FLR and FOW effects (solid line, circles) is compared with the PIC growth rate resulting from the simulations without the fast-particle FLR effects (dotted line, squares). The hybrid-MHD [34, 35] growth rate (dashed line, no symbols) is shown, too. For other parameters see the main text. 

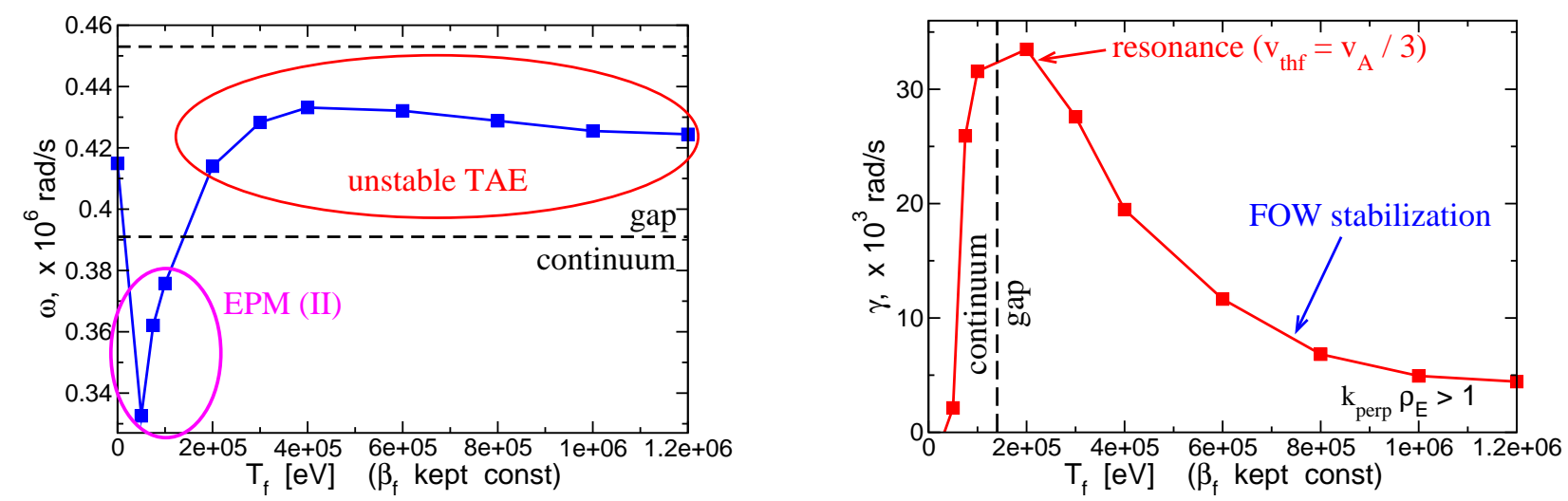

FIG. 7: (Color online) The frequency (left) and the growth rate (right) of the TAE mode destabilized by the fast particles (sweep over the temperature of the fast particles, $\beta_{f}=0.134 \%$ is kept constant). EPM instability appears at the lower temperatures, where the fast particles resonantly interact with the bottom part of the continuum. For parameters see the main text. 

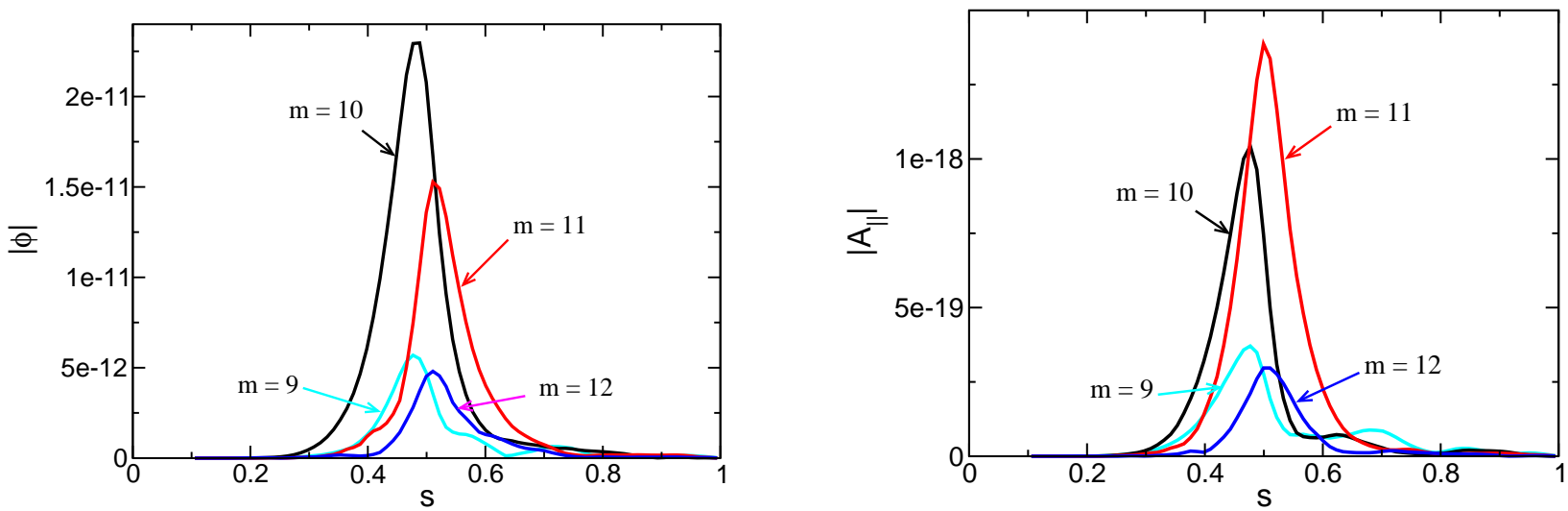

FIG. 8: (Color online) Radial structures of the electrostatic (left) and magnetic (right) potentials measured with the PIC code (for $T_{f}=0.6 \mathrm{MeV}$, see Fig. 7). The mode is centered in the position of the gap and has a TAE-type poloidal spectrum (with the dominant poloidal modes $m=10$ and $m=11)$. 

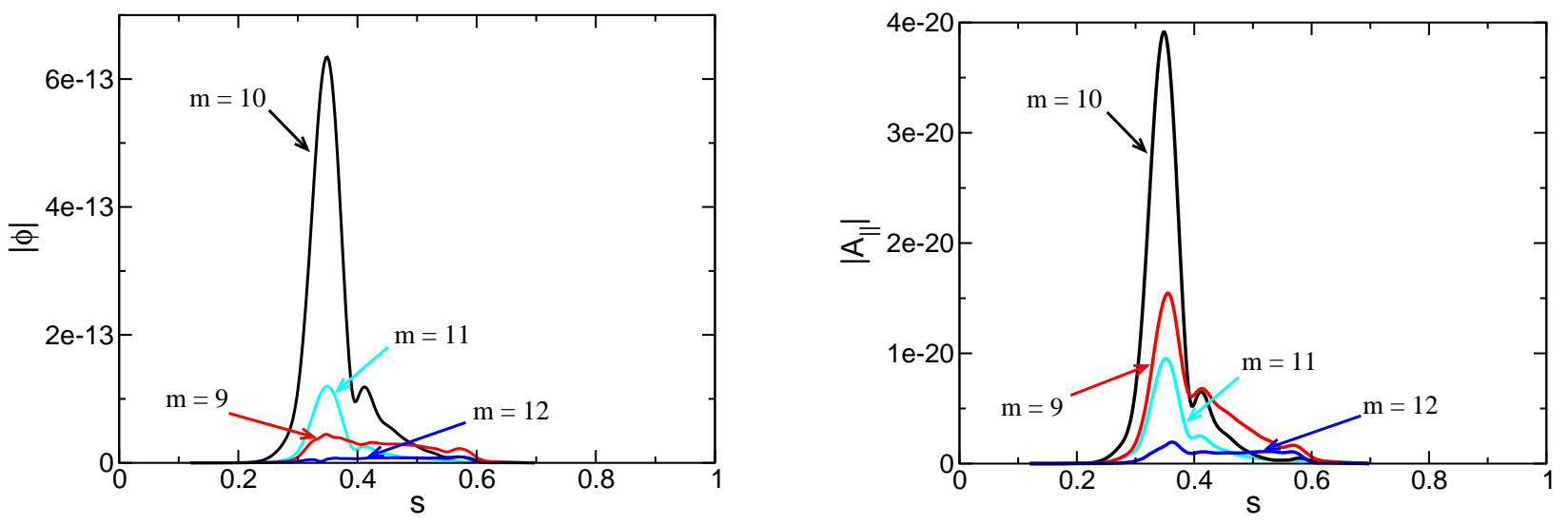

FIG. 9: (Color online) Radial structures of the electrostatic (left) and magnetic (right) potentials measured with the PIC code (for $T_{f}=50 \mathrm{keV}$, see Fig. 7). The mode is shifted radially from the gap position. A single poloidal mode $m=10$ dominates. This is a non-preturbative EPM mode. 

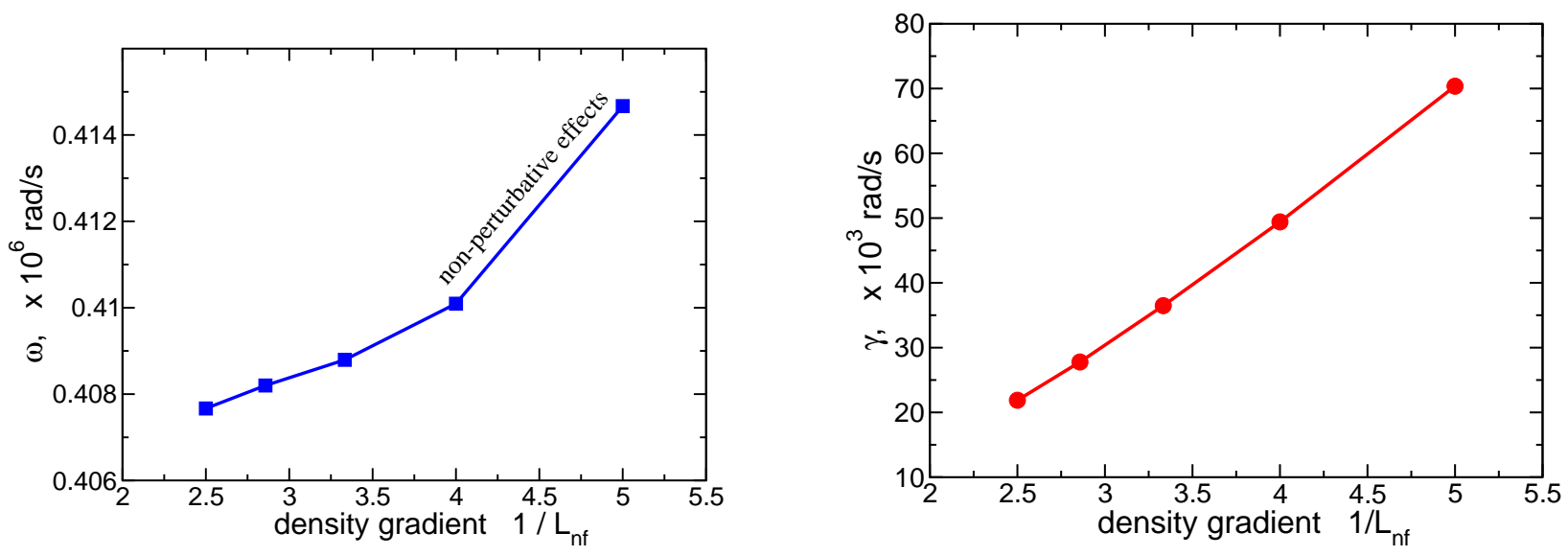

FIG. 10: (Color online) The frequency (left) and the growth rate (right) of the TAE mode (sweep over the fast-particle density gradient). The fast-particle temperature $T_{f 0}=0.2 \mathrm{MeV}$, the fast particle density $n_{f 0}=1.5 \times 10^{17} \mathrm{~m}^{-3}$. For other parameters see the main text. 

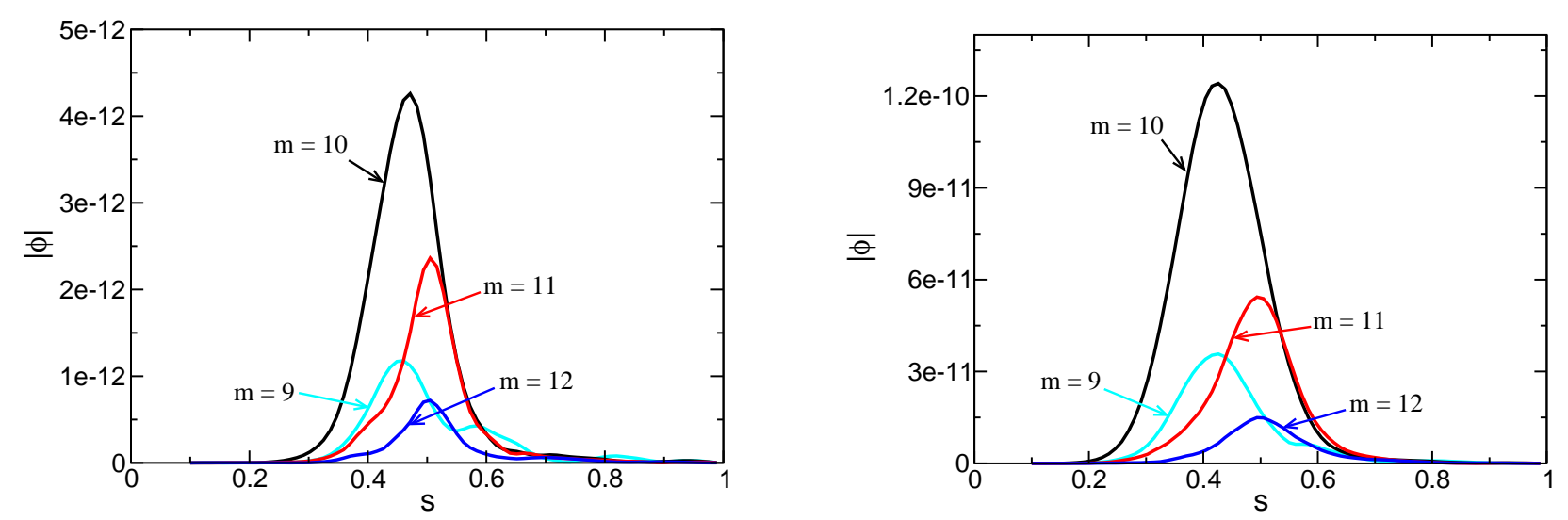

FIG. 11: (Color online) The radial structure of the electrostatic potential obtained with the PIC code. The figure on the left corresponds to $1 / L_{\mathrm{n} f}=2.5$. The figure on the right results from the simulations with $1 / L_{\mathrm{n} f}=5.0$. Other parameters in these two simulations coincide. 

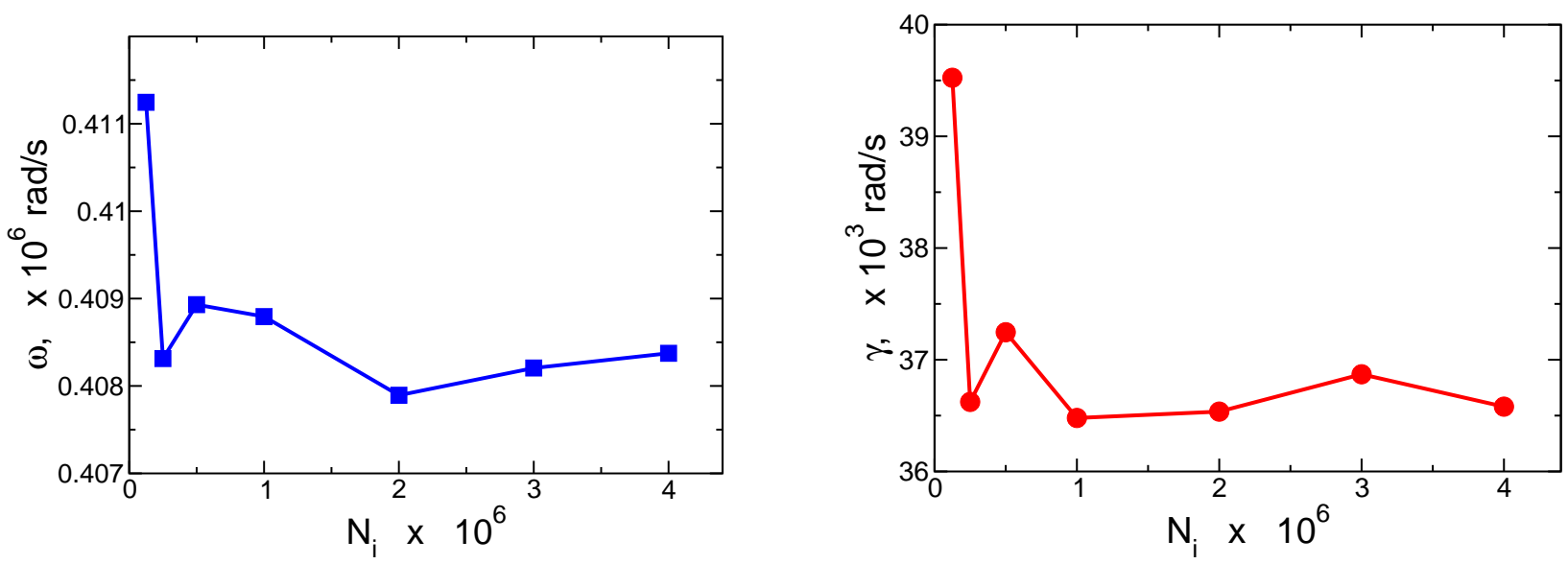

FIG. 12: (Color online) The frequency (left) and the growth rate (right) of the TAE mode destabilized by the fast particles (convergence study with respect to the marker resolution). The fastparticle temperature $T_{f 0}=0.2 \mathrm{MeV}$, the fast particle density $n_{f 0}=1.5 \times 10^{17} \mathrm{~m}^{-3}$. For other parameters see the main text. 

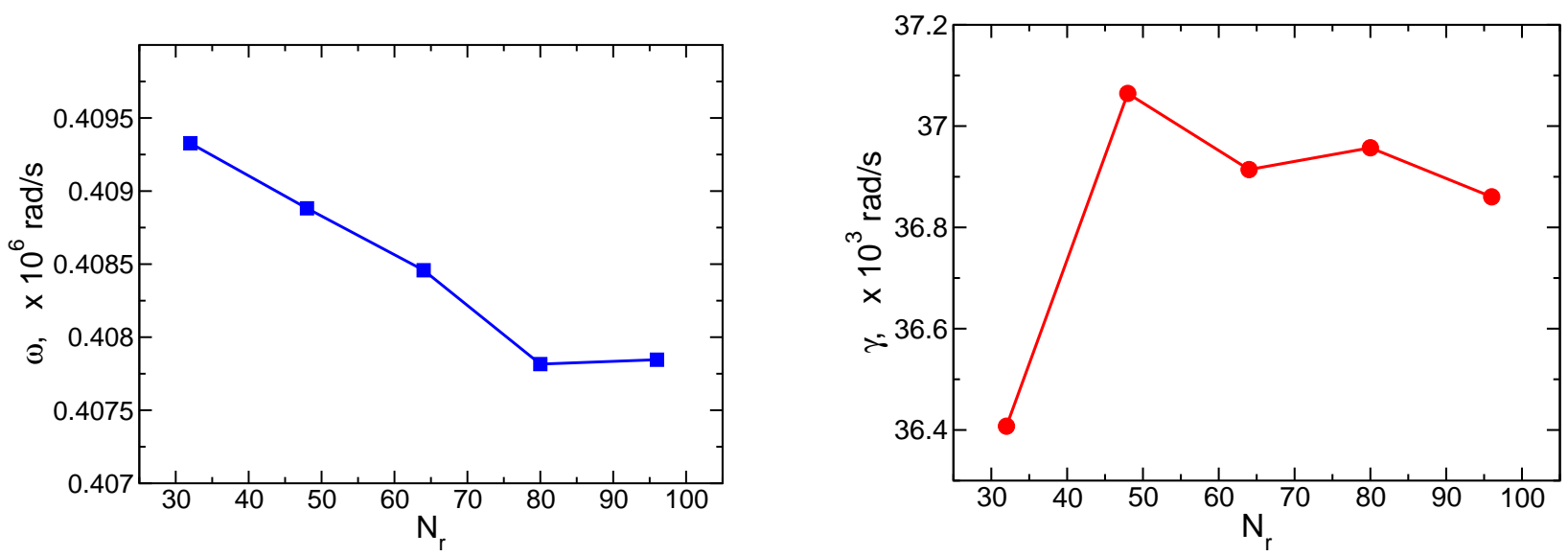

FIG. 13: (Color online) The frequency (left) and the growth rate (right) of the TAE mode destabilized by the fast particles (convergence study with respect to the radial grid resolution). The fast-particle temperature $T_{f 0}=0.2 \mathrm{MeV}$, the fast particle density $n_{f 0}=1.5 \times 10^{17} \mathrm{~m}^{-3}$. For other parameters see the main text. 

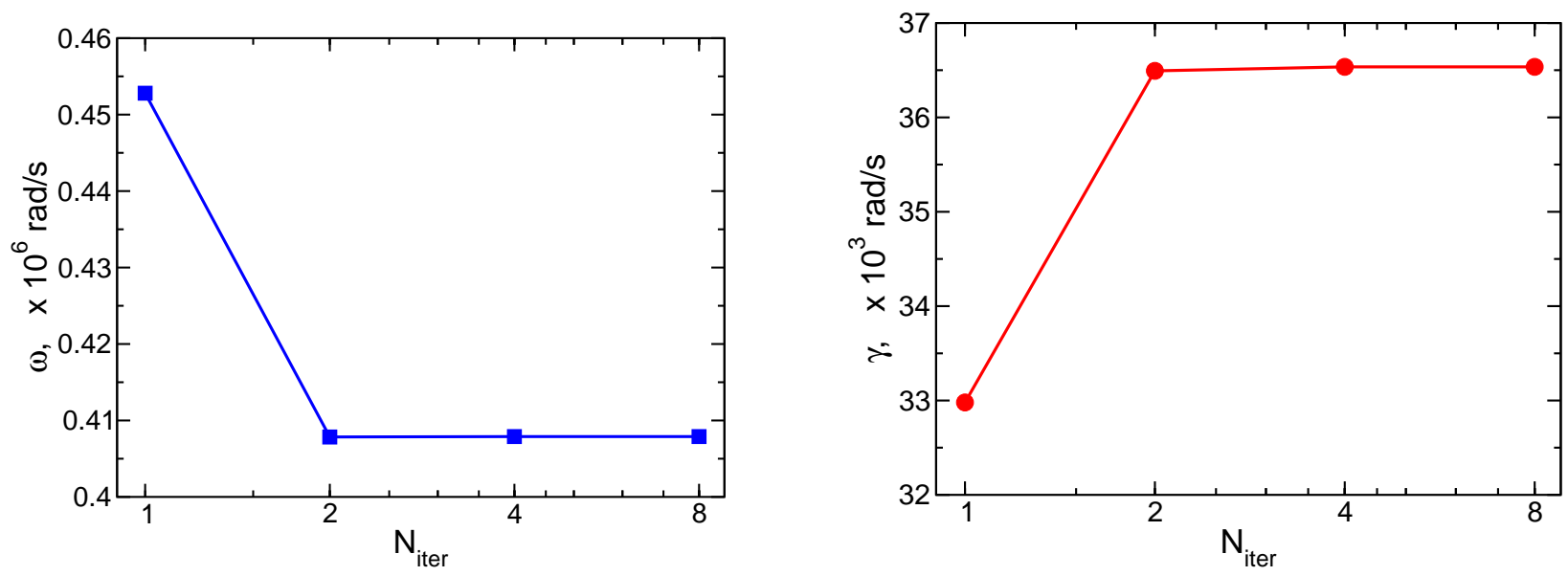

FIG. 14: (Color online) The frequency (left) and the growth rate (right) of the TAE mode destabilized by the fast particles (convergence study with respect to the number of iterations in Ampére's law). In this simulation, $N_{i}=2 \times 10^{6}$ ion markers, $N_{e}=8 \times 10^{6}$ electron markers and $N_{f}=4 \times 10^{6}$ fast-particle markers have been taken. The radial resolution is $N_{r}=80$. The fast-particle temperature $T_{f 0}=0.2 \mathrm{MeV}$, the fast particle density $n_{f 0}=1.5 \times 10^{17} \mathrm{~m}^{-3}$. 